\title{
Flow Simulation of Gas Cyclone Separator at High Reynolds Number Using the Elliptic-Relaxation Hybrid LES/RANS (ER-HRL) Model
}

\author{
Mohamed A. Sayed ${ }^{1,2}$, Abdel Dehbi ${ }^{2}$, Bojan Niceno ${ }^{2}$, Konstantin Mikityuk ${ }^{1,2}$, Maria Krinner ${ }^{3}$ \\ ${ }^{1}$ Paul Scherrer Institut \\ 5232 Villigen PSI, Switzerland \\ Mohamed.sayed@psi.ch; Abdel.dehbi@psi.ch; Bojan.Niceno@psi.ch \\ ${ }^{2}$ Swiss Federal Institute of Technology Lausanne (EPFL) \\ 1015 Lausanne, Switzerland \\ Konstantin.mikityuk@psi.ch \\ ${ }^{3}$ Swiss Federal Institute of Technology Zurich (ETHZ) \\ 8092 Zürich, Switzerland \\ Krmaria@ethz.ch
}

\begin{abstract}
In this paper, we investigate Wall-Modeled Large Eddy Simulation (WMLES) in a stairmand high-efficiency cyclone separator at two Reynolds numbers i.e. $\mathrm{Re}=33,045-280,000$. We compute the gas flow using the elliptic relaxation hybrid RANS/LES (ER-HRL). The model employs a wall integration linear eddy viscosity RANS model for the wall-adjacent region, and switches to the LES dynamic model in the bulk flow. At the lower Reynolds number i.e. $R e=33,045$, we investigate the effect of varying cone bottom opening - rendering three different cyclone configurations - on cyclone performance. Flow statistics are reported at several locations across the cyclone axis where both mean and RMS values are observed. For high Reynolds number i.e. $\operatorname{Re}=280,000$, results are compared against LES and experimental databases from literature. Model predictions of mean flow are in good agreement with reference data, while higher-order moments i.e. RMS values are not very well predicted by the model despite following the same trend of experimental data. Results are in a global good agreement with LES and experimental data at a fraction of well-resolved LES CPU cost. This analysis will serve as a good basis for further investigation of cyclone grade efficiency using Lagrangian particle tracking.
\end{abstract}

Keywords: CFD; Cyclone separator; Stairmand high-efficiency cyclone; hybrid LES/RANS; wall-modeled LES.

\section{Introduction}

Over the last few decades, reversed flow cyclones have been the most widely used dust separators in many industrial areas, from agro and pharma services to mining and petrochemical sectors. Despite its simple design concept, the performance optimization and flow dynamics of a cyclone separator are exceedingly complicated [1]. In order to overcome this complexity, computational fluid dynamics (CFD) simulations have been used to assess the performance of cyclone separators giving a comprehensive inspection of the flow field [5-18]. The first cyclone CFD-based predictions go back to the early numerical simulations of a turbulent flow field in 1982 [2].

In principle, cyclone performance can be assessed using Lagrangian particle tracking. This, in the first place, requires an accurate prediction for the underlying carrier fluid flow. Many endeavors have been made to predict the flow in gas cyclones using Reynolds-Averaged Navier Stokes (RANS) turbulence models. In particular, Reynolds Stress Model (RSM) and the RNG $\kappa-\varepsilon$ have been investigated with scrutiny alongside Lagrangian particle tracking (LPT) to study cyclone collection efficiency $[5-14 ; 19-33]$. The swirling flow in such high-efficiency cyclones implies naturally prominent anisotropic effects at the wall vicinity. It was reported that the RSM model is well suited to handling such strong anisotropic effects [12-13; 34-35]. However, it was shown in several studies that the RSM is unable to accurately predict higher-order moments i.e. RMS velocity values [17, 29, 34, 36-38]. It is also worth mentioning that standard linear eddy viscosity (LEV) RANS models fail to predict the correct mean axial or tangential velocity profiles. This was outlined in the works of Hoekstra et al, 2000 [4], and Kaya et al, 2008 [39] by comparing the RNG $\kappa-\varepsilon$ model results with the experimental data. Unlike RANS, LES gives a time-dependent solution with much fewer modeling errors. However, LES simulation can be very expensive, especially in the case of high Reynolds numbers and concurrent simulations between gas flow and discrete particles. 
To the best of our knowledge, no cyclone separator study has been conducted before using the hybrid LES/RANS approach. The rationale behind this approach is to engage RANS mode near the wall (where mesh resolution can be stringent) and switch to LES in the outer flow region. This method proved to substantially reduce the required computer power relative to traditional LES without considerably compromising the accuracy [41]. In this paper, we use the elliptic relaxation hybrid RANS/LES (ER-HRL) model to investigate the swirling flow inside cyclone separator.

\section{Methodology}

In this section, the methodology for solving the gas flow is presented. Both mass and momentum conservation equations are solved with the elliptic relaxation (ER-HRL) as a hybrid RANS/LES turbulence model. In this study, we use T-Flows [42], which is a second-order accurate, unstructured, cell-centered, finite volume, in-house CFD code. The code was validated for many turbulent flow benchmarks in both canonical and complex flows [42 - 45].

$$
\begin{gathered}
\frac{\partial u_{i}}{\partial t}+u_{j} \frac{\partial u_{i}}{\partial x_{j}}=-\frac{1}{\rho} \frac{\partial p}{\partial x_{i}}+\frac{v \partial^{2} u_{i}}{\partial x_{i} \partial x_{j}} \\
\frac{\partial u_{i}}{\partial x_{i}}=0
\end{gathered}
$$

We use the Linear Eddy Viscosity (LEV) approximation to close the Reynolds stress term that stems from averaging the non-linear advection term in the momentum conservation equation Eq. (1). Using the Boussinesq eddy viscosity assumption [46], the stress tensor is evaluated by the space-filtered velocity field, which is linked to the mean velocity gradients. Using this hypothesis, the Reynolds stress tensor reads:

$$
\tau_{i j}=-\overline{u_{i} u_{j}}=v_{t}\left(\frac{\partial \bar{u}_{i}}{\partial x_{j}}+\frac{\partial \bar{u}_{j}}{\partial x_{i}}\right)-\frac{2}{3} k \delta_{i j}
$$

where $\mathrm{k}$ is the turbulent kinetic energy (TKE), and $\delta_{i j}$ is the Kronecker delta. The subgrid-scale (SGS) eddy viscosity, $\mathrm{v}_{t}$ is then computed as the maximum of RANS and LES eddy viscosity values (as in Eq. 4). This serves as the first switching criterion between LES and RANS modes.

$$
\begin{gathered}
\mathrm{v}_{t}=\max \left(\mathrm{v}_{t, R A N S}, \mathrm{v}_{t, L E S}\right) \\
\mathrm{v}_{t, R A N S}=C_{\mu} k \tau \xi \\
\mathrm{v}_{t, L E S}=\left(\Delta_{L E S}\right)^{2} C_{d y n}|S|
\end{gathered}
$$

Above $C_{d y n}$ is the dynamic Smagorinsky constant, and $\Delta_{L E S}$ is the classical definition of LES cut-off length (the cubic root of cell volume), and $|S|=\sqrt{2 S_{i j} S_{i j}}$ is the magnitude of the strain rate, $S_{i j}$. The four-equation RANS model was first proposed by M. Hadziabic [48] to mitigate the scaling of computational cost with Reynolds number in turbulent wallbounded flows. The model was recently tested in complex industrial configurations, capturing with high accuracy the Eulerian statistics at high turbulence levels using relatively coarse meshes [42]. The model offers a second switching criterion which ensures a fast LES/RANS passage. This is accomplished through the blending function, $\alpha$ (Eq. 13) which is incorporated in the TKE transport equation (Eq. 7), $\mathrm{k}$ to re-scale the dissipation rate, $\varepsilon$. In the near-wall region, the model 
acts in unsteady RANS (URANS) mode (where $\alpha=1$ ), while away from the wall, alpha becomes larger than 1 since the characteristic length from the RANS energy-containing range becomes more prominent (Eq. 14). This results in damping of the TKE (Eq. 7), and hence diminishing $v_{t, R A N S}$ (Eq. 5), which in turn activates the LES mode through Eq. 4. The RANS mode in this model is a four-equation model that transports the quantities $k-\varepsilon-\xi-F$ (Equations 7-10). This RANS model was shown to have a high level of robustness in a number of benchmarks [47]. For the LES mode, the Smagorinsky dynamic model is activated, which predicts the correct eddy viscosity needed to damp fluctuations in the near-wall region [48]. It was also noted by [42] that the backscatter of the dynamic LES subgrid-scale model ensures a smooth transition across the RANS-LES interface, which substantially mitigates the log-layer mismatch anomaly.

$$
\frac{D k}{D t}=P_{k}-\alpha \varepsilon+\nabla \cdot\left(\left(v+v_{t}\right) \nabla k\right)
$$

Where $P_{k}=v_{t} S_{i j} S_{i j}$ is the production of TKE. The dissipation rate transport equation reads

$$
\frac{D \varepsilon}{D t}=\frac{C_{\varepsilon 1} P_{k}-C_{\varepsilon 2} \varepsilon}{\tau}+\nabla \cdot\left(\left(v+v_{t}\right) \nabla \varepsilon\right)
$$

Derived from $v^{2}$ and $\mathrm{k}$ equations in Durbin [49], the transport equation of the normalized wall-normal stress reads

$$
\frac{D \xi}{D t}=F-\frac{\varepsilon}{k} P_{k}+\nabla \cdot\left(\left(v+v_{t}\right) \nabla \xi\right)
$$

The elliptic-relaxation equation is then introduced to account for the inviscid wall-blocking effects with time and length scale limiters, $\tau$ and $\mathrm{L}$ as shown below

$$
\begin{gathered}
L^{2} \nabla^{2} F-F=\frac{1}{\tau}\left(c_{1}+C_{2}^{\prime} \frac{P_{k}}{\varepsilon}\right)\left(\xi-\frac{2}{3}\right) \\
\tau=\max \left[\min \left(\frac{k}{\varepsilon}, \frac{a}{\sqrt{6} C_{\mu} \xi|S|}\right), C_{\tau}\left(\frac{v}{\varepsilon}\right)^{1 / 2}\right] \\
L=C_{L}\left(\max \left[\min \left(\frac{k^{2 / 3}}{\varepsilon}, \frac{k^{1 / 2}}{\sqrt{6} C_{\mu} \xi|S|}\right), C_{\eta}\left(\frac{v^{3}}{\varepsilon}\right)^{1 / 4}\right]\right) \\
\alpha=\max \left(1, \frac{L_{R A N S}}{L_{L E S}}\right) \\
L_{R A N S}=\frac{k^{3 / 2}}{\varepsilon}, \quad L_{L E S}=C_{\alpha}(\Delta X . \Delta Y . \Delta Z)^{1 / 3}
\end{gathered}
$$

Model parameters were fixed in all simulations, and the values used are shown below in Table 1 for reproducibility. 
Table 1: Model parameters

\begin{tabular}{|c|c|c|c|c|c|c|c|c|c|}
\hline $\mathrm{C} \mu$ & $C_{\varepsilon 1}$ & $C_{\varepsilon 2}$ & $C_{1}$ & $C_{2}^{\prime}$ & $C_{\tau}$ & $C_{L}$ & $\mathrm{a}$ & $C_{\eta}$ & $C_{\alpha}$ \\
\hline 0.22 & $1.4(1+0.012 / \xi)$ & 1.9 & 0.4 & 0.65 & 6.0 & 0.36 & 0.6 & 85 & 0.8 \\
\hline
\end{tabular}

As shown in Table 2, we consider three cyclone configurations based on cyclone bottom opening. Inlet velocity is set to have a uniform profile, the outlet is set as convective outflow boundary, and the no-slip condition is imposed for walls. For the pressure velocity coupling, we use the SIMPLE (semi-implicit method pressure-linked equations) algorithm, and for momentum equations' discretization, the MinMod scheme is used. The time step size was prescribed to achieve a timeaccurate solution with the maximum Courant number $(\mathrm{CFL}) \leq 1$ throughout all the simulations. Mass density is set to $1.205 \mathrm{~kg} / \mathrm{m}^{3}$ while molecular viscosity is $1.82 \mathrm{e}-5 \mathrm{~kg} \cdot \mathrm{m}^{-1} \cdot \mathrm{s}^{-1}$. SnappyHexMesh tool in OpenFOAM v6 code was used to construct 325211-element mesh. To have a one-to-one comparison with LES and experimental databases [3, 4, 40], we probe mean and RMS velocity profiles at three positions across the cyclone axis (as in Fig. 1) as a function of the barrel diameter. Distances are measured from the dustbin entrance as position $1,1.015 \mathrm{D}_{\mathrm{c}}$; position $2,1.16 \mathrm{D}_{\mathrm{c}}$ : position $3,1.52 \mathrm{D}_{\mathrm{c}}$ (i.e. $\mathrm{y}_{1}=$ $0.031456 \mathrm{~m}, \mathrm{y} 2=0.03596 \mathrm{~m}, \mathrm{y} 3=0.04712 \mathrm{~m}$ ). It is worth mentioning that the flow inside the cyclone geometry is naturally unstable due to anisotropic and swirling effects. This makes it quite challenging for the solver to converge the flow. For this sake, we recommend using a standard RANS model first to develop the flow across the cyclone axis. The model can then be swiftly switched from the RANS to the desired hybrid turbulence model once (at least) one flow-through time is achieved.

Table 2: Cyclone design parameters

\begin{tabular}{|l|c|c|c|}
\hline Design parameters $(\mathrm{m})$ & Cyclone 1 & Cyclone 2 & Cyclone 3 \\
\hline Body diameter, Dc & 0.031 & 0.031 & 0.031 \\
\hline Gas outlet diameter, De & 0.0155 & 0.0155 & 0.0155 \\
\hline Inlet height, a & 0.0125 & 0.0125 & 0.0125 \\
\hline Inlet width, b & 0.005 & 0.005 & 0.005 \\
\hline Cyclone height, H & 0.077 & 0.077 & 0.077 \\
\hline Cylinder height, h & 0.031 & 0.031 & 0.031 \\
\hline Gas outlet duct length, S & 0.0155 & 0.0155 & 0.0155 \\
\hline Vortex finder extension, V & 0.0325 & 0.0325 & 0.0325 \\
\hline Cone bottom opening, B & 0.0194 & 0.0116 & 0.005 \\
\hline Bin collector height, C & 0.03 & 0.03 & 0.03 \\
\hline
\end{tabular}

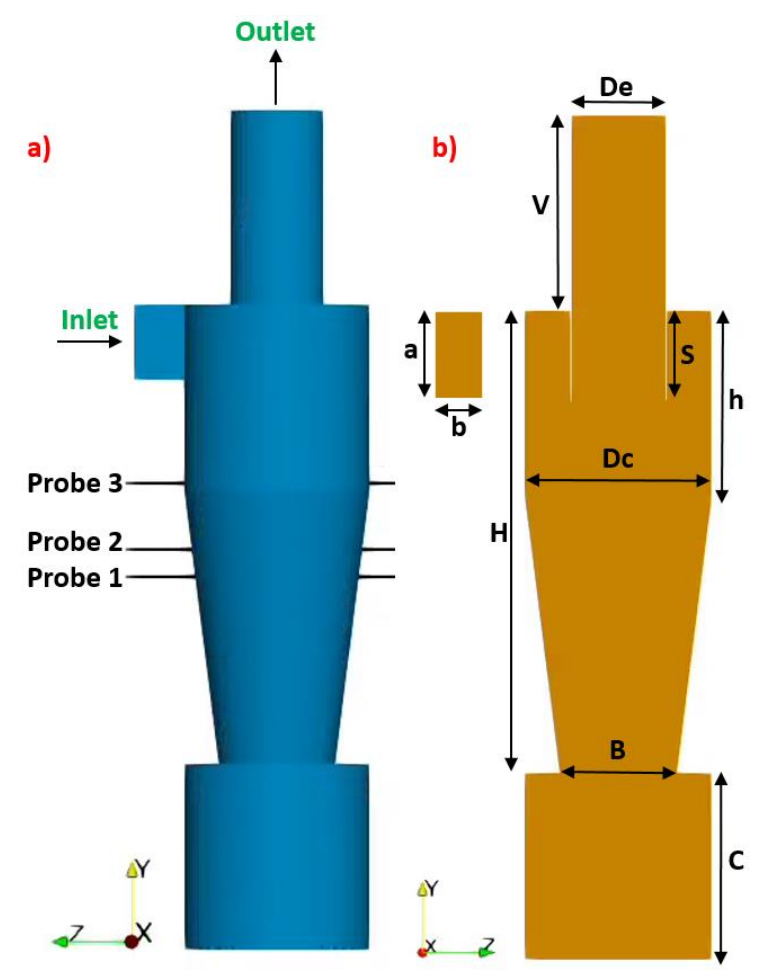

Fig. 1: a) Velocity probes, b) cyclone parameter

\section{Results}

In this section, we show the results of the fluid flow simulation at both Reynolds numbers i.e. $\operatorname{Re}=33,045-280,000$ based on cyclone barrel diameter and inlet velocity $\left(\mathrm{Re}=\mathrm{U}_{\mathrm{in}} \mathrm{D}_{\mathrm{c}} / \mathrm{v}\right)$. It must be noted that in the case of higher Reynolds number, the geometry was up-scaled by a factor of 9.36 to stay in the incompressible flow zone (Mach number $<0.3$ ). 


\section{1. $R e=34,045$}

To analyze the sensitivity of cyclone performance to the bottom cone-diameter, we show the results of the three cyclones at relatively low turbulence level i.e. $\mathrm{Re}=34,045$. As shown in Figure 2 below, Mean and RMS velocity profiles are plotted at the three probe locations mentioned above. Turbulence statistics are gathered after 40 units of integral timescale i.e. $\tau_{I}=$ $D_{c} / U_{\text {in }}$ over a time interval of another 40 time units, where the flow could be assumed statistically stationary. As shown in Fig. 3-a, mean and RMS tangential velocities increase when decreasing the cone bottom diameter, B which is due to mass conservation. It is also noticed that the tangential velocity profiles almost do not change when decreasing the cone bottom diameter below $0.0116 \mathrm{~m}$. This is not surprising since the mass flow rate going through the collector bin is decreasing until it vanishes when dimension B nullifies. At this point, the swirling mass flow as a whole will go up towards the vortex finder upon reaching the bottom of the cyclone cone. On the other hand, looking at the axial mean velocity profiles, we see that cyclone 1 and 3 are rather following the same trend, while for RMS values we observe that cyclone 2 and 3 profiles have the same behavior at the cyclone barrel section (position 3).

a) Mean
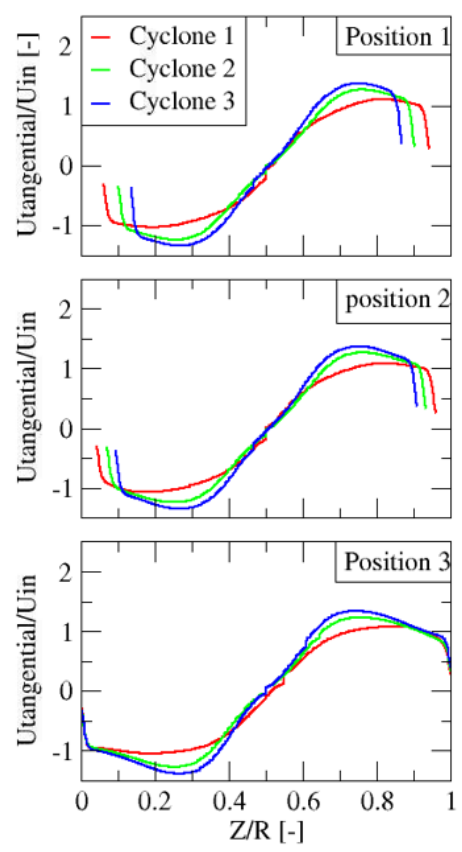

RMS
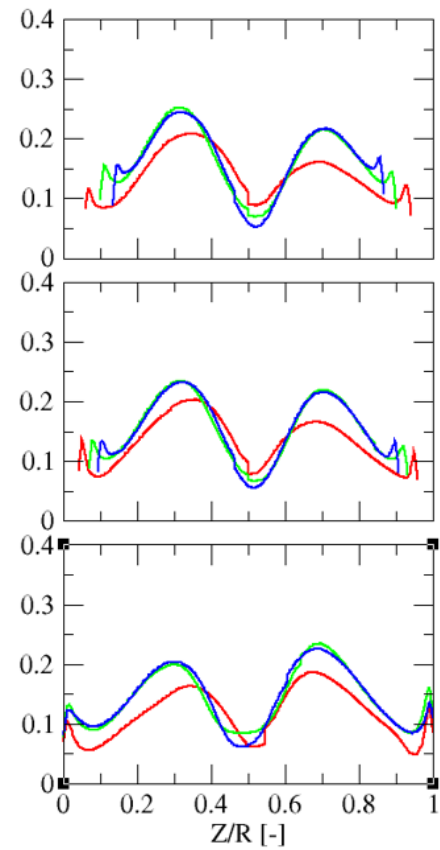

b)
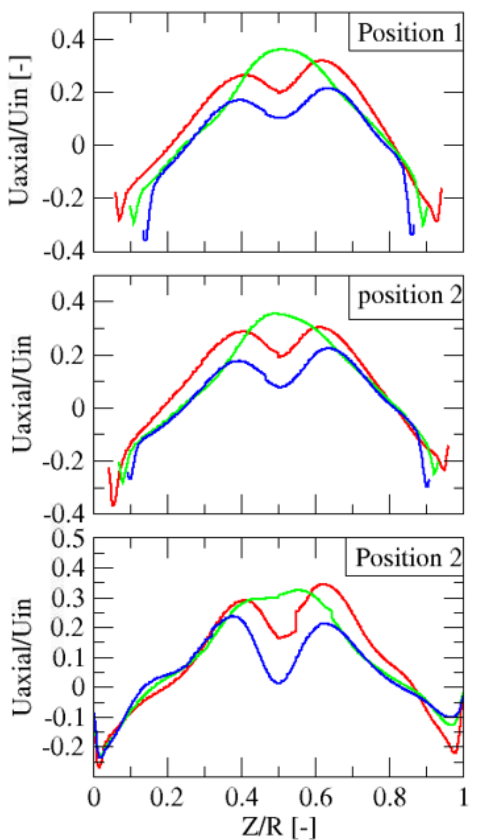

RMS

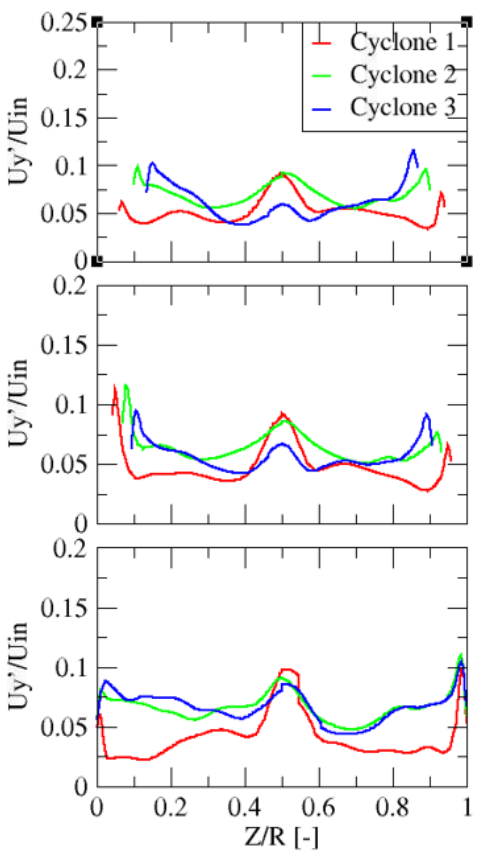

Fig. 2: Mean and RMS velocity profiles; a) tangential component left, b) axial component right. Velocities are computed for each cyclone at different locations at $\mathrm{Re}=33,045$. Results are reported for the ER-HRL model.

\section{2. $\operatorname{Re}=280,000$}

In this section, we show the results of the ER-HRL model predictions at approximately one order of magnitude higher Reynolds number. Results are compared against each of the well-resolved LES data from Derksen, 2003 [3], LES data by Jose et al, 2012 [40], and the experimental data by Hoekstra, 2000 [4]. It was verified that the ER-HRL model is running in active wall-modeled LES mode throughout the simulation. This was observed by computing the eddy to molecular viscosity ratio along with the three measuring probes, where recorded values were between 25-55. From Fig. 3a-b, it can be noticed that the model predicts the mean flow globally. However, the peak of the mean tangential velocity is slightly underpredicted as in Fig. 3-a. On the other hand, it could be noticed that RMS values are overpredicted for the tangential velocity (Fig. 3a) and underpredicted for the axial component (Fig. 3b). Nonetheless, RMS values still agree with the same trend as the experimental data. The deviation in results from pure LES data could be attributed to the model incapability of proper treatment to the boundary layer in such a highly anisotropic swirling flow. 

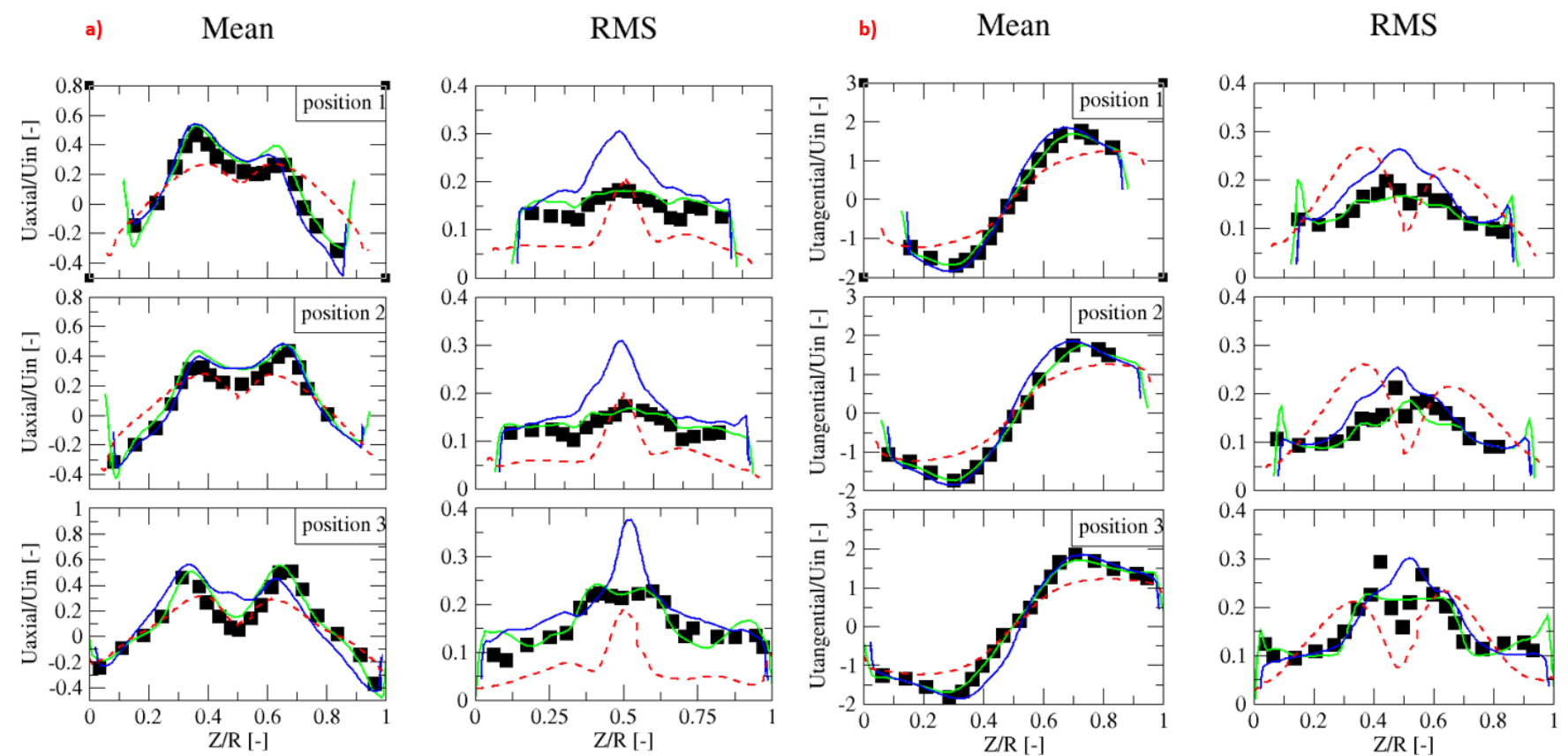

Fig. 3: Mean and RMS velocity profiles at $\mathrm{Re}=280,000$; a) Axial component (left), b) Tangential component (right). Black symbols refer to the experimental data by Hoekstra [3], blue and green lines represent LES results from Jose et al, 2012 and Derksen respectively [40,3], and red dashed lines are own results from the ER-HRL model for cyclone 1.

\section{Conclusion}

In this study, the ER-HRL model was investigated in a stairmand high-efficiency gas cyclone at two Reynolds number limits i.e. $\operatorname{Re}=33,045-280,000$. For the lower Reynolds number, three cyclone models based on the bottom cone diameter were compared to study the sensitivity of flow statistics to cyclone design. For the high Reynolds number, we compare model predictions to LES and experimental databases from literature. Mean and RMS velocity profiles are reported at different locations across cyclone axis. Results show that the model is able to predict the mean flow using relatively very coarse mesh. Although RMS values are following the same trend of the experiment, axial RMS velocity profiles were shown to be slightly underpredicted, while the RMS values of the tangential component are quite overpredicted. Given that the swirling flow in this benchmark is highly anisotropic at the wall region, authors argue that this discrepancy could be attributed to the deficiency of RANS models based on linear eddy viscosity models in handling swirling flows with strong anisotropic effects. This was also noticed in previous studies [4, 39]. Based on the good results obtained by Jose et al, 2012 [40] with a relatively coarse mesh, it is worth investigating in the following steps to test the model in LES dynamic mode with no active wall modeling. This investigation will be important for the next step of the ongoing research - studying cyclone separation efficiency using Lagrangian particle tracking.

\section{Acknowledgements}

This research project is sponsored by Swiss National Science Foundation (SNSF) under grant number [200021175532] in membership with the Federal pension fund PUBLICA according to the regulation of ETH domain (VR-ETH 1). The CPU hours needed to accomplish this work are provided by Merlin6 HPC cluster at Paul Scherrer Institut in Switzerland.

\section{References}

[1] A.C. Hoffman, L.E. Stein, Gas Cyclones and Swirl Tubes: Principles, Design and Operation, Springer, 2008.

[2] Boysan, F., W. H. Ayers, and J. Swithenbank, "A Fundamental Mathematical Modelling Approach to Cyclone Design," Trans. Inst. Chem. Engrs., 60, 222 Ž1982. 
[3] J.J. Derksen, Separation performance predictions of a stairmand high-efficiency cyclone, AIChE Journal 49 (2003) 13591371

[4] A.J. Hoekstra, "Gas flow field and collection efficiency of cyclone separators", PhD thesis, Technische Universiteit Delft, 2000.

[5] T.G. Chuah, J. Gimbun, T.S.Y. Choong, A CFD study of the effect of cone dimensions on sampling aerocyclones performance and hydrodynamics, Powder Technology 162 (2006) 126-132.

[6] M. Azadi, M. Azadi, A. Mohebbi, A CFD study of the effect of cyclone size on its performance parameters, Journal of Hazardous Materials 182 (2010) 835-841.

[7] A. Raoufi, M. Shams, M. Farzaneh, R. Ebrahimi, Numerical simulation and optimization of fluid flow in a cyclone vortex finder, Chemical Engineering and Processing 47 (2008) 128-137.

[8] K.U. Bhaskar, Y.R. Murthy, M.R. Raju, S. Tiwari, J.K. Srivastava, N. Ramakrishnan, CFD simulation and experimental validation studies on hydrocyclone, Minerals Engineering 20 (2007) 60-71.

[9] S. Schuetz, G. Mayer, M. Bierdel, M. Piesche, Investigations on the flow and separation behavior of hydrocyclones using computational fluid dynamics, International Journal of Mineral Processing 73 (2004) 229-237.

[10] L. Shi, D. Bayless, Comparison of boundary conditions for predicting the collection efficiency of cyclones, Powder Technology 173 (2007) 29-37.

[11] G. Wan, G. Sun, X. Xue, M. Shi, Solids concentration simulation of different size particles in a cyclone separator, Powder Technology 183 (2008). 940-194.

[12] J. Jiao, Y. Zheng, G. Sun, J. Wang, Study of the separation efficiency and the flow field of a dynamic cyclone, Separation and Purification Technology 49 (2006) 157-166.

[13] B. Wang, D.L. Xu, K.W. Chu, A.B. Yu, Numerical study of gas-solid flow in a cyclone separator, Applied Mathematical Modelling 30 (2006) 1326-1342.

[14] A. Kepa, Divison of outlet flow in a cyclone vortex finder-the CFD calculations, Separation and Purification Technology 75 (2010) 127-131.

[15] A.J. Hoekstra, J.J. Derksen, H.E.A. van den Akker, An experimental and numerical study of turbulent swirling flow in gas cyclones, Chemical Engineering Science 54 (1999) 2055-2065.

[16] J.J. Derksen, Separation performance predictions of a stairmand high-efficiency cyclone, AIChE Journal 49 (2003) $1359-1371$.

[17] J.J. Derksen, H.E.A. van den Akker, S. Sundaresan, Two-way coupled large-Eddy simulations of the gas-solid flow in cyclone separators, American Institute of Chemical Engineers 54 (2008) 872-884.

[18] J.J. Derksen, S. Sundaresan, H.E.A. van den Akker, H.E.A., Simulation of mass- loading effects in gas-solid cyclone separators, Powder Technology 163 (2006) 59-68.

[19] W.D. Griffiths, F. Boysan, Computational fluid dynamics (CFD) and empirical modelling of the performance of a number of cyclone samplers, Journal of Aerosol Science 27 (1996) 281-304.

[20] L. Ma, D.B. Ingham, X. Wen, Numerical modelling of the fluid and particle penetration through small sampling cyclones, Journal of Aerosol Science 31 (2000) 1097-1119.

[21] J. Gimbun, T.G. Chuah, T.S.Y. Choong, A. Fakhru'l-Razi, Prediction of the effects of cone tip diameter on the cyclone performance, Aerosol Science 36 (2005) 1056-1065.

[22] S. Bernardo, M. Mori, A.P. Peres, R.P. Dionísio, 3-D computational fluid dynamics for gas and gas-particle flows in a cyclone with different inlet section angles, Powder Technology 162 (2006) 190-200.

[23] B. Zhao, Y. Su, J. Zhang, Simulation of gas flow pattern and separation efficiency in cyclone with conventional single and spiral double inlet configuration, Chemical Engineering Research and Design 84 (2006) 1158-1165.

[24] M. Narasimha, M.S. Brennan, P.N. Holtham, T.J. Napier-Munn, A comprehensive CFD model of dense medium cyclone performance, Minerals Engineering 20 (2007) 414-426.

[25] K. Elsayed, C. Lacor, The effect of cyclone inlet dimensions on the flow pattern and performance, Applied Mathematical Modelling 35 (2011) 1952-1968. 
[26] K. Elsayed, C. Lacor, Modeling, analysis and optimization of aircyclones using artificial neural network, response surface methodology and CFD simulation approaches, Powder Technology 212 (2011) 115-133.

[27] D. Masoud, M.S. Shahidzadeh, A CFD study of the effect of vortex finder thickness on gas cyclone separator performance, Journal of Applied Sciences 11 (2011) 2570-2577.

[28] H. Safikhani, M. Shams, S. Dashti, Numerical simulation of square cyclones in small sizes, Advanced Powder Technology 22 (2011) 359-365.

[29] S.K. Shukla, P. Shukla, P. Ghosh, Evaluation of numerical schemes for dispersed phase modeling of cyclone separators, Engineering Applications of Computational Fluid Mechanics 5 (2011) 235-246.

[30] Y. Su, A. Zheng, B. Zhao, Numerical simulation of the effect of the inlet configuration on square cyclone separator performance, Powder Technology 210 (2011) 293-303.

[31] R.M. Valverde, J.R. Coury, J.A.S. Gonçalves, Numerical Modeling of Particle Dynamics in a Cyclone Separator, 21 st Brazilian Congress of Mechanical Engineering, October 24-28, 2011, Natal, RN, Brazil.

[32] K. Elsayed, C. Lacor, Modeling and pareto optimization of gas cyclone separator performance using RBF type artificial neural networks and genetic algorithms, Powder Technology 217 (2012) 84-99.

[33] Y. Qiu, B. Deng, C.N. Kim, Numerical study of the flow field and separation efficiency of a divergent cyclone, Powder Technology 217 (2012) 231-237.

[34] M.D. Slack, R.O. Prasad, F. Boysan, Advances in cyclone modeling using unstructured grids, Institution of Chemical Engineers 78 (2000) 1098-1104.

[35] S.K. Shukla, P. Shukla, P. Ghosh, Evaluation of numerical schemes using different simulation methods for the continuous phase modeling of cyclone separators, Advanced Powder Technology 22 (2011) 209-219.

[36] W.P. Martignoni, S. Bernardo, L. Quintani, Evaluation of cyclone geometry and its influence on performance parameters by computational fluid dynamics (CFD), Brazilian Journal of Chemical Engineering 24 (2007) 83-94.

[37] G. Gronald, J.J. Derksen, Simulating turbulent swirling flow in a gas cyclone: a comparison of various modelling approaches, Powder Technology 205 (2011) 160-171.

[38] G.I. Pisarev, A.C. Hoffmann, W. Peng, H.A. Dijkstra, Large Eddy Simulation of the vortex end in reverse flow centrifugal separators, Applied Mathematics and Computation 217 (2011) 5016-5022.

[39] F. Kaya, I. Karagoz, Performance analysis of numerical schemes in highly swirling flows in cyclones, Current Science 94 (2008) 1273-1278.

[40] Francisco José de Souza, Ricardo de Vasconcelos Salvo, Diego Alves de Moro Martins, Large Eddy Simulation of the gas-particle flow in cyclone separators, Separation and Purification Technology 94 (2012) 61-70.

[41] Hadziabdic, M., Hanjalic, K., 2020. Elliptic-relaxation hybrid rans-les (ERHRL) for complex wall-bounded fluid and heat flows, ERCOFTAC Bulletin 121.

[42] Niceno, B., Hanjalic, K., e.a., 2001. T-flows. Available on Github: https://github.com/DelNov/T-Flows.git/.

[43] Niceno, B, 2005. PhD thesis, Unstructured large eddy and conjugate heat transfer simulations of wall-bounded flows.

[44] Palkin, E., Hadžiabdiф, M., Mullyadzhanov, R., Hanjaliф, K., 2018. Control of flow around a cylinder by rotary oscillations at a high subcritical Reynolds number. Journal of Fluid Mechanics 855.

[45] Hadžiabdiф, M., Hanjaliф, K., 2008. Vortical structures and heat transfer in a round impinging jet. Journal of Fluid Mechanics 596.

[46] Boussinesq, J., 1877. Essai sur la théorie des eaux courantes. Caporaloni, M., T.F.T.F..V.O., 1975. Transfer of particles in nonisotropic air turbulence. Journal of Atmospheric Sciences.

[47] Hanjaliф, K., Popovac, M., Hadžiabdiф, M., 2004. A robust near-wall elliptic-relaxation eddy-viscosity turbulence model for cfd. International Journal of Heat and Fluid Flow 25. doi:10.1016/j.ijheatfluidflow.2004.07.005. Horn, M., S.

[48] Hadžiabdic, M., 2006. Les, rans and combined simulation of impinging flows and heat transfer. PhD thesis, doi: http://resolver.tudelft.nl/uuid:47cb00a2-d935-4a0a-b68b-a0440cfb9d26.

[49] Durbin, P.A., 1991. Near-wall turbulence closure modeling without "damping functions". Theoretical and Computational Fluid Dynamics 3. 